\title{
A Review Extended Release Oral Drug Delivery System and Multiparticulate Drug Delivery Systems (MDDS)
}

\author{
Swapnil B. Khambat*, Shubham A. Kale \\ R. C. Patel Institute of Pharmaceutical Education and Research.Shirpur, Dhule, Maharashtra, India \\ Corresponding Author E-mail: swapnilkhambat777@gmail.com
}

\begin{abstract}
The extended release product will optimize therapeutic effect and safety of a drug at the same time improving the patient convenience and compliance. By incorporating the dose for $24 \mathrm{hrs}$ into one tablet/capsule from which the drug is released slowly. The concept of multiple unit dosage form was initially introduced in the early 1950's.These forms play a major role in the design of solid dosage form processes because of their unique properties and the flexibility found in their manufacture. These forms can be defined as oral dosage forms consisting of a multiplicity of small discrete units, each exhibiting some desired characteristics. The release of drug from pellets depends on a variety of factors including the carrier used to form pellets and the amount of drug contained in them. Consequently, pellets provide tremendous opportunities for designing new controlled and extended release oral formulations, thus extending the frontier of future pharmaceutical development. The possible mechanism for drug release includes solution/diffusion through the continuous polymer phase or plasticizer channels, diffusion through aqueous pores and osmotically driven release through aqueous pores. To distinguish between these mechanisms, the release rate was studied as a function of coating thickness, plasticizer content and osmotic pressure in the dissolution medium.
\end{abstract}

Keywords : Extended Release, Oral Route, Therapeutic Concentration, Pellet, Dosage Form

\section{INTRODUCTION}

Extended release formulations make the drug available over extended time period after oral administration. The extended release product will optimize therapeutic effect and safety of a drug at the same time improving the patient convenience and compliance. By incorporating the dose for $24 \mathrm{hrs}$ into one tablet/capsule from which the drug is released slowly. This formulation helps to avoid the side effects associated with low and high concentrations. The ideal drug delivery system should show a constant zero-order release rate and maintain the constant plasma concentrations. ${ }^{1-3}$

\section{Advantages ${ }^{2,3}$}

Extended release products having many advantages.

a) The extended release formulations may maintain therapeutic concentrations over prolonged periods.

b) The use of extended release formulations avoids the high blood concentration.

c) Extended release formulations have the potential to improve the patient compliance.

d) Reduce the toxicity by slowing drug absorption.

e) Increase the stability by protecting the drug from hydrolysis or other degradative changes in gastrointestinal tract.

f) Minimize the local and systemic side effects. 
g) Improvement in treatment efficacy.

h) Minimize drug accumulation with chronic dosing.

i) Usage of less total drug.

j) Improvement the bioavailability of some drugs.

k) Improvement of the ability to provide special effects.

\section{METHODS AND MATERIAL}

Drug properties, which are suitable for, extended release formulation ${ }^{2,3}$

a) Physiochemical Properties of the drug.

1. Aqueous solubility: $(>0.1 \mathrm{mg} / \mathrm{ml})$

2. Partition co-efficient: (1000:1 octanol: water system)

3. Drug stability in vivo: (High enough, so drug remain stable during release from system)

4. Protein binding: (Drug with high protein binding will not required release modification)

5. Drug pKa \& ionization at physiological $\mathrm{pH}$ : (pKa for acidic API= 3.0 - 7.5, pKa for Basic API = 7.0 11.0) 6. Mechanisms and sites of absorption: (Mechanism of absorption should not be active type and absorption window should not be narrow)

6. Molecular size and diffusivity: (Molecule size should be small (100-400 D so it can be easily diffused through polymer matrix)

7. Dose size: $(<300 \mathrm{mg})$

\section{b) Biological Properties of Drug}

1. Distribution: (A.P.I. with large volume of distribution is not suitable).

2. Metabolism: (A.P.I. should be metabolized with intermediate speed).

3. Half-life of drug: $(2-8 \mathrm{hrs})$.

4. Margin of safety: (High enough so dose dumping does not cause any serious side effect).
5. Plasma concentration response relationship: (A.P.I. having linear relationship is better candidate).

\section{Disadvantages of Extended Release Delivery System. ${ }^{4}$}

1. Extended release formulation contains a higher drug load and thus any loss of integrity of the release characteristics of the dosage form.

2. The larger size of extended release products may cause difficulties in ingestion or transit through gut.

3. The release rates are affected by various factors such as food and the rate of transit through the gut.

4. Some differences in the release rate from one dose to another dose but these have been minimized by modern formulations.

5. High cost of preparation.

6. Sometimes the target tissue will be exposed to constant amount of drug over extended period results in drug tolerance.

\section{Multiparticulate Drug Delivery Systems(MDDS)}

The concept of multiple unit dosage form was initially introduced in the early 1950's. These forms play a major role in the design of solid dosage form processes because of their unique properties and the flexibility found in their manufacture. These forms can be defined as oral dosage forms consisting of a multiplicity of small discrete units, each exhibiting some desired characteristics. Together, these characteristics units provide the overall desired controlled release of the dose. These multiple units are also referred to as pellets, spherical granules or spheroids. Pelletization is an agglomeration process that converts fine powders or granules of bulk drugs and excipients into small, free flowing, spherical or semi-spherical units, referred to as pellets. Pellets or spherical granules are produced by agglomerating fine 
powders with a binder solution. These pellets usually range in size from $0.5-1.5 \mathrm{~mm}$ and in applications may be as large as $3 \mathrm{~mm}$. In MDDS, drug substances are divided into number of subunits, typically consist of thousands of spherical particles having diameter of about $0.05-2.00 \mathrm{~mm}$. To administer or to recommend total dose these subunits are compressed into a tablets or filled into a sachets or encapsulated. ${ }^{5}$ The formulation of multicomponent MDDS is also possible because it shows different mechanism of action, provides additive/ synergistic effect, and reduces the doses of individual agents and limited side effects. Though it is costlier than monotherapies in short term, it reduces treatment failure rate, lower case fatality ratios and reduction in development of resistance for development of new products in long term therapy. ${ }^{6}$

\section{ADVANTAGES OF MULTIPARTICULATES (PELLETS)}

The use of pellets as a vehicle for drug delivery at controlled rate has recently received significant attention. Pellets can be prepared by many methods, the drug-layering technique is most widely used today. Multiparticulates provide various advantages ${ }^{3}$ as given below

1. Avoidance of the dose dumping

2. Gastric emptying is faster.

3. Performance is less dependent on nutritional state as multiparticulates are sufficiently small and can be evacuated through pylorus during digestive phase.

4. Shows improved reproducibility of transit time and high degree of dispersion in digestive tract.

5. Better distributed and less likely to cause local irritation.

6. Improved stability, patient comfort and compliance.

7. Achieve unique release pattern.
8. Extend patent protection, globalize product and overcome competition.

\section{DRAWBACKS OF MULTIPARTICULATES (PELLETS)}

1. Low drug loading

2. Proportionally higher need for exciepients

3. Lack of manufacturing reproducibility and efficacy

4. Large number of process variables

5. Multiple formulation steps

6. Higher cost of production

7. Need of advanced technology

8. Trained/skilled personal needed for manufacturing

\section{VARIOUS TECHNIQUES OF PELLETIZATION}
A. Powder layering technique
B. Solution / suspension layering technique
C. Extrusion- spheronization technique
D. Balling / spherical agglomeration
E. Spray congealing / drying
F. Cryopelletization
G. Melt spheronization

A. Powder Layering: Various steps involved in this technique are as follows:

1. Sifting/milling

2. Loading of non pareil seeds

3. Drug coating

4. Drying

5. Sizing

6. Functional coating

7. Encapsulation

Converting powders to pellets can be achieved by a variety of techniques. Layering a suspension or solution of drug onto a seed material can result in pellets that are uniform in size distribution and 
generally possess very good surface morphology. These characteristics are especially desirable when the pellets will subsequently be coated for some type of controlled release for $24 \mathrm{~h}$. This method involves the disposition of successive layers of solutions and/or suspensions of drug substances and binder on starter seeds, which may be inert materials or granules of the same drug. In principle, the factors that control coating processes apply to solution or suspension layering and as a result, require basically the same processing equipments. In the initial stages, the drug particles are bound to the starter seeds and subsequently to the forming pellets with the help of liquid bridges originated from the sprayed liquid. These liquid bridges are eventually replaced by solid bridges derived either from a binder in the application medium or from any other material, including the drug substances, that is soluble in the liquid. Successive layering of the drug and binder solution continues until the desired pellet size is reached. Throughout the process, it is extremely important to deliver the powder accurately at a predetermined rate and in a manner that maintains equilibrium between the binder liquid application rate and the powder delivery rate. If the powder delivery rate is not maintained at predetermined equilibrium levels, over wetting or dust generation may occur and neither the quality nor the yield of the product can be maximized. Towards the end of the layering process, it is likely that fines may be generated owing to potential inter particle and wallto-particle friction and appears in the final product thereby lowering the yield. The problem can be overcome if the application medium is sprayed on the cascading pellets at the end of the layering process to increase the moisture level at the pellet surface and facilate layering of the fines onto the pellets. The equipments like tangentianal spray equipment, centrifugal fluid bed granulator, rotary granulator are used for this purpose. ${ }^{7}$

\section{B. Solution / suspension layering technique}

Various steps involved in this technique are as follows:

1. Mixing/milling

2. Loading of non pareil seeds

3. Drug coating

4. Drying

5. Sizing

6. Functional coating

7. Encapsulation

This method involves the deposition of successive layers of solution and/or suspensions of drug substances and binders on starter seeds, which may be inert materials or crystals/granules of the same drug. The factors that control coating processes apply to solution or suspension layering and require basically the same processing equipment. Consequently, conventional coating pan, fluid bed granulator and wruster coaters have been used successfully to manufacture pellets. The wruster coating process which was invented about 30 years ago, had evolved through elaborate design modifications and refinement into ideal equipment for the manufacture of pellets by solution/suspension layering. The high drying efficiency inherent in fluid bed equipment, coupled with the innovative and efficient design features of the Wruster process, has allowed the machines to hold center stage in pharmaceutical processing technology. The disadvantage of the Wruster process is the inaccessibility of the nozzle. If the nozzles are clogged at any time during the layering process, the operation has to be interrupted and the spray guns must be removed for cleaning. The problem can be alleviated by screening the formulation or by using a spray gun with a bigger nozzle. Another aspect of the process that is challenging when multiple nozzles are used is the potential overlap of adjacent spray zones. Although the position of the nozzle is fixed, the spray zone 
overlap can be minimized using the air cap at the end of the spray gun.

During processing, all the components of the formulations are first dissolved or suspended in an appropriate quantity of application medium to provide a formulation with the desired viscosity and is then sprayed onto the product bed. The sprayed droplets immediately impinge on the started seeds and spread evenly on the surface, provided the drying conditions and fluid dynamics are favourable. This is followed by a drying phase that renders dissolved materials to precipitate and form solid bridges that would hold the formulation components tighter as successive layers on the started seeds. The process continues until the desired quantity of drug substance and thus target potency of the pellets is achieved. Ideally, no new nuclei are formed, and the particle population remains the same. However, the sizes of the pellets increase as a function of time, and as a result, the total mass of the system also increases. Optimization of process variables is difficult for the successful development of a palletized product. ${ }^{8}$

\section{Extrusion and spheronization}

Various steps involved in this technique are as follows: Sifting/milling

Mixing/binding

Extrusion

Spheronization

Drying

Sizing

Coating

Encapsulation

This is a multistep process involving dry mixing, wet granulation, extrusion, spheronization, drying and screening. The first step is dry mixing of the drug and excipients in suitable mixers followed by wet granulation, in which the powder is converted in to a plastic mass that, can be easily extruded. The extruded strands are transferred into a Spheronizer, where they are instantaneously broken into short cylindrical rods on contact with the rotating friction plate and pushed outward and up the stationary wall of the processing chamber by centrifugal force. Finally, owing to gravity, the particles fall back to the friction plate, and the cycle is repeated until the desired sphericity is achieved. The technology is unique in that it is not only suitable for manufacture of pellets high drug loading but it also can be used to produce extended-release pellets in certain situations in a single step and thus can obviate the need for subsequent film coating. Extrusion - spheronization is a multistep process involving a number of unit operations and equipment. However, the most critical pieces of processing equipment that, in effect, dictate the outcome of overall process are the extruders and spheronizer. ${ }^{9}$

A variety of extruders, which differ in design features and operational principles are currently on the market and can be classified as screw-fed extruders, gravity-fed extruders, and ram extruders. Screw-fed extruders have screws that rotate along the horizontal axis and hence transport the material horizontally. They may be axial or radial screw extruders. Axial extruders, which have a die plate that is positioned axially, consist of a feeding zone, a compression zone and an extrusion zone. The product temperature is controlled during extrusion by jacketed barrels. In radial extruders, the transport zone is short and the material is extruded radically through screens mounted around the horizontal axis of the screws.

Gravity-fed extruders include the rotary cylinder and rotary gear extruders which differ primarily in the design of the two counter-rotating cylinders. In the rotary-cylinders extruder, one of the two counterrotating cylinders is hollow and perforated, whereas the other cylinder is solid and cats as a pressure roller. In the so-called rotary-gear extruder, there are two 
hollow counter-rotating gear cylinders with counter bored holes. In ram extruders, a piston displaces and forces the material through a die at the end. Ram extruders are preferred during formulation development they are designed to allow for measurement of the rheological properties of formulation. In an extrusion - spheronization process, formulation components such as filler, lubricants and $\mathrm{pH}$ modifiers play a critical role in producing pellets with the desired attributes. The granulated mass must be plastic and sufficiently cohesive and self lubricating during extrusion. During the spheronization step, it is essential that the extrudates break at appropriate length and have sufficient surface moisture to enhance formation of uniform spherical pellets. ${ }^{10}$

\section{Solution and Suspension Layering ${ }^{11}$}

Principle of the suspension and solution layering process: Solution and suspension layering involve the deposition of successive layers of solutions and suspensions of drug substances, respectively, on starter seeds that may be inert materials or crystals or granules of the same drug. In principle, the factors that control coating processes apply directly to solution or suspension layering. During solution or suspension layering, all the components of the formulation are dissolved or suspended in the application medium and hence determine the solids contents and the viscosity of the liquid sprayed. As the solution or suspension is sprayed onto the product bed, the droplets impinge on the starter seeds or cores and spread evenly on the surface, provided that the drying conditions and fluid dynamics are favorable. This is followed by the drying phase which allows dissolved materials to crystallize and form solid bridges between the core and initial layer of the drug substance as well as among the successive layers of drug substance. The process continues until the desired layers of drug and hence the target potency of the pellets are achieved. The rate of particle growth is rather slow due to the incremental addition of the dissolved or suspended drug .In this process, though the particle population remains the same, the size of the pellets increases as a function of time and, as a result, the total mass of the system increases.

\section{E. Cryopelltization}

This is the process whereby droplets of liquid formulations are converted into solid spherical particles or pellets by using liquid nitrogen as fixing medium. The technology which was initially developed for lyophilization of viscous bacterial suspension can be used to produce drug-loaded pellets in liquid nitrogen at $160^{\circ} \mathrm{C}$. The procedure permits instantaneous and uniform freezing of the processed material owing to the rapid heat transfer that occurs between the droplets and thus the large surface area facilate the drying process. The amount of liquid nitrogen required for manufacturing a given quantity depends on the solids content and temperature of the solution or suspension being processed. It is usually between 3 and $5 \mathrm{~kg}$ per kilogram of finished pellets.

\section{F. Melt spheronization}

It is a process whereby a drug substance and excipients are converted into a molten or semi molten state and subsequently shaped using appropriate equipment to provide solid spheres or pellets. The drug is blended with the excipients, polymers and waxes and extruded at predetermined temp. The extrusion temp must be high enough to melt at least one of the components. The extrudates is cut into uniform cylindrical segments with a cutter and then they are spheronized resulting pellets are dried. ${ }^{12,13}$

\section{RESULTS AND DISCUSSION}

\section{CHARACTERIZATION OF PELLETS 14-21 Crushing strength}


Strength testing was performed in 20 pellets of each formulation with an available radial force apparatus.

\section{Density and porosity}

Densities were derived as follows: An exact quantity ' $\mathrm{M}$ ' of pellets was taken and was placed into a measuring cylinder. Volume ' $\mathrm{V}$ ' occupied by the pellets was noted without disturbing the cylinder and bulk density was calculated using the following equation

\section{Bulk density $=\mathrm{M} / \mathrm{V}$}

The tapping method was used to determine the tapped density in which the cylinder containing known amount $(\mathrm{M})$ of pellets was subjected to a fixed number of taps (approximately 100) until the bed of pellets had reached the minimum. The final volume after tapping 'Vo ' was recorded and the tap density was calculated by the following equation:

\section{Tapped density $=\mathrm{M} / \mathrm{V}_{0}$}

Angle of repose, Hausner ratio and Carr index (\% compressibility index) were determined to predict flowability. A higher Hausner ratio indicates greater cohesion between pellets while a high Carr index is indicative of the tendency to form bridges. Angle of repose of the pellets is the maximum angle possible between the surface of the pile of pellets and the horizontal plane was obtained by fixed funnel method using the formula:

$$
\begin{aligned}
& \tan \theta=h / r \\
& h=\text { height of pile } \\
& r=\text { radius of pile }
\end{aligned}
$$

Where, $\mathrm{h}$ is height and $\mathrm{d}$ is the diameter of the pallets pile that is on a paper after making the pallets flow from the glass funnel.

\section{Hausner ratio and Carr's index}

Hausner ratio and Carr's index were calculated using the formulae:

$\%$ compressibility index $=$ Tapped density - Bulk density $\mathbf{x} 100$

\section{Tapped density}

Here, V and Vo are the volumes of the sample before and after the standard tapping respectively and $C$ is Carr index.

\section{In vitro dissolution studies}

Dissolution was conducted in a USP (Method 1, rotating basket) apparatus, at a speed of $100 \mathrm{rpm}$, in $900 \mathrm{ml}$ of dissolution media (phosphate buffer at $\mathrm{pH}$ $7.2 \pm 0.05)$, maintained at $37 \pm 0.5^{\circ} \mathrm{C}$, using an automated assembly UV spectrophotometer.

\section{Stability study of sustained release pellets}

Stability study of pellets was performed as at room temperature, $40^{\circ} \mathrm{C} / 75 \% \mathrm{RH} \& 30^{\circ} \mathrm{C} / 70 \% \mathrm{RH}$ for three months. The physical properties of pellets as well as the In vitro release profile of the drug was found to be a function of the different storage conditions as well as the physico-chemical nature of the polymers.

\section{IV.CONCLUSION}

There are several reasons for attractiveness of these dosage forms: provides increased bioavailability of drug product reduction in the frequency of administration to prolong duration of effective blood levels reduces the fluctuation of peak trough concentration and side effects and possibly improves the specific distribution of the drug. If one were to develop an ideal drug delivery system, two prerequisites would be required: Firstly single dose for the duration of treatment whether for days or weeks as with infection. Second it should deliver the active entity directly to the site of action minimizing the side effects. Drug delivery systems keeping in mind 
the toxic effects of the drugs incorporated and to maintain the overall stability of the product on the account of the properties of natural polymers. Thus MDDS with natural polymer.The review extended release oral drug delivery system and multiparticulate drug delivery systems(MDDS) various techniques of pelletization and characterization of pellets.

\section{ACKNOWLEDGEMENT}

Authors are highly Acknowledge the help of friend of R.C.Patel Institute of Pharmaceutical Education and Research.Shirpur. For providing necessary information required for review work. Also we are highly Acknowledge the help and guidance of Mrs.Shubham A.Kale

\section{REFERENCES}

[1]. Pfizer, Detrol ${ }^{\circledR}$ L.A. tolterodine tartrate extended release capsule,

http://www.drugbank.ca/system/fda_labels/ DB01036.pdf). Accessed October 2011.

[2]. Ehab Ahmed MH. Ph.D. Thesis: Application and evaluation of extended release technology to loop diuretics. University of Cincinnati (2002): 78-82.

[3]. Madhusudhan P and Nazeer S: Extended release formulation. International Journal of Pharmacy and Technology 2010; 2: 625- 684.

[4]. Hayashi T. Formulation, study and drug release mechanism of a new Theophylline sustained release preparation, Int. J Pharm., 2005; 304: 91101.

[5]. Shaji J, Chadawar V and Talwalkar P:Multiparticulate Drug Delivery System. The Indian Pharmacist. 2007;6(60):21-28.

[6]. Dashora K, Saraf S and Saraf S. Development of sustained release multicomponent microparticulate system of Diclofenac sodium and Tizanidine hydrochloride. Int J Pharm Sci and nanotechnology. 2008;1:98-105.

[7]. Jamila Hamdani, Andre' J. Moe"s and Karim Amighi, 2002. Development and evaluation of prolonged release pellets obtained by the melt pelletization process. International Journal of Pharmaceutics, 245, 167-177.

[8]. Vinayak D Kadam and Surendra G Gattani, 2009. Effect of Curing Time on $\mathrm{pH}$ and Time Dependant Coated Pellets. International Journal of Health Research, 2(1), 75-81.

[9]. F. Podczeck, P.E. Knight and J.M. Newton, 2008. The evaluation of modified microcrystalline cellulose for the preparation of pellets with high drug loading by extrusion/spheronization. International Journal of Pharmaceutics, 350, 145-154.

[10]. Srujan Reddy, Palash Das, Harika Das and Arpita Ghosh, 2011. MUPS (Multiple Unit Pellet System) Tablets - A Brief Review. Journal of Pharmaceutical and Biomedical Sci., 12(02), 1-5.

[11]. Thoma $\mathrm{K}$ and Ziegler I: The $\mathrm{pH}$ independent release of fenoldopam from pellets with insoluble film coats. Eur J Pharm and Biopharm. 1998;46(1):105-113.

[12]. Nikolett Kállai, Oliver Luhn, Judit Dredán, Kristóf Kovács, Miléna Lengyel and István Antal, 2010. Evaluation of drug release from coated pellets based on isomalt, sugar and microcrystalline cellulose inert cores. AAPS Pharm. Sci.Tech., 1(1), 383-391.

[13]. Guanhao Ye, Siling Wang, Paul Wan Sia Heng, Ling Chen and Chao Wang, 2007. Development and optimization of solid dispersion containing pellets of Itraconazole prepared by high shear pelletization. International Journal of Pharmaceutics, 337, 80-87.

[14]. C.P. Jain and P.S. Naruka, 2009. Formulation and evaluation of fast dissolving tablets of Valsartan. International Journal of Pharmacy and Pharmaceutical Sciences. 1(1), 219- 226. 
[15]. Dhirendra Kumar, Vivek Dave, Shaila Lewis, Brajesh Parmar, Kavita R. Gajbhiye, Sarvesh Paliwal, 2010. Design and evaluation of sustained-release matrix once daily formulation of Stavudine. International Journal of Drug Delivery, 2, 125-134.

[16]. Gihan Nabil Fetih, 2010. Formulation and characterization of Gelucire pellets for sustain release of Ibuprofen. Bull. Pharm. Sci., Assiut University, 33(2), 217-224.

[17]. Golam Kibria, KM Ariful Islam and Reza-Ul Jalil, 2009. Stability studies of Ambroxol Hydrochloride sustain release pellets coated with acrylic polymer. Pak. J. Pharm. Sci., 22(1), 36-43.

[18].V. S. N. Murthy Dwibhashyam and J. Vijayaratana, 2008. Key formulation variables in tableting of coated pellets. Indian Journal of Pharmaceutical Sciences, 70 (5), 555-564.

[19]. Jaber Emami, Mona Tajeddin and Fatemeh Ahmadi, 2008. Preparation and In vitro evaluation of sustained-release matrix tablets of Flutamide using synthetic and naturally occurring polymers. Iranian Journal of Pharmaceutical Research, 7 (4), 247-257.

[20]. Phutane P, Shidhaye S, Lotlikar V, Ghule A, Sutar S and Kadam V, 2010. In vitro evaluation of novel sustained release microspheres of Glipizide prepared by the emulsion solvent diffusion-evaporation method. J Young Pharmacists, 2, 35-41.

[21]. Subal Chandra Basak, Kesevan Senthil Kumar and Murugesan Ramalingam, 2008. Design and release characteristics of sustained release tablet containing Metformin $\mathrm{HCl}$. Brazilian Journal of Pharmaceutical Sciences, 44(3), 477-483.

\section{Cite this article as :}

Swapnil B. Khambat, Shubham A. Kale., "A Review Extended Release Oral Drug Delivery System and Multiparticulate Drug Delivery Systems (MDDS)", International Journal of Scientific Research in Science and Technology (IJSRST), Online ISSN : 2395-602X, Print ISSN : 2395-6011, Volume 7 Issue 1, pp. 84-92, January-February 2020. Available at doi : https://doi.org/10.32628/IJSRST207124

Journal URL : http://ijsrst.com/IJSRST207124 